\title{
Archéopages
}

Archéopages

Archéologie et société

Hors-série 2 | 2010

Archéologie sans frontières

\section{Collaboration franco-israélienne dans la recherche archéologique}

Hamoudi Khalaily

\section{(2) OpenEdition}

1 Journals

Édition électronique

URL : https://journals.openedition.org/archeopages/806

DOI : 10.4000/archeopages.806

ISSN : 2269-9872

Éditeur

INRAP - Institut national de recherches archéologiques préventives

Édition imprimée

Date de publication : 1 octobre 2010

Pagination : 113-115

ISSN : 1622-8545

\section{Référence électronique}

Hamoudi Khalaily, «Collaboration franco-israélienne dans la recherche archéologique », Archéopages

[En ligne], Hors-série 2 | 2010, mis en ligne le 01 octobre 2010, consulté le 23 février 2023. URL : http:// journals.openedition.org/archeopages/806 ; DOI : https://doi.org/10.4000/archeopages.806

Tous droits réservés 
blanche a protégé les vestiges archéologiques dans les parties non fouillées de la nécropole.

L'étude archéo-anthropologique de cette nécropole vise surtout à redonner voix «aux plus nombreux » : l'objectif était de définir l'attitude du groupe inhumant vis-à-vis des défunts dont il s'est occupé avec plus ou moins de précaution, voire d'ostentation. Or les interventions archéologiques précédentes n'ont laissé aucune information de ce type, pas même sous la forme rudimentaire de documents photographiques. Cette nécropole, comme bien d'autres, a en effet largement souffert d'une approche archéologique centrée sur l'objet. De telle sorte que, comme l'a souvent dit Henri Duday, on pourrait croire que le squelette, rarement enregistré, souvent laissé in situ à Plinthine, accompagnait les offrandes... et non l'inverse ! Tant il est vrai que la découverte d'un squelette a souvent été considérée comme une gêne, l'objet prenant alors le pas sur le défunt ou ce qu'il en reste. La description et le prélèvement des squelettes ont donc été orientés selon les modalités de l'archéo-thanatologie funéraire (Duday, 2005; Duday, 2006; Duday, Guillon, 2006). Comme pour les opérations préventives en France, cela revient à reconnaître les distorsions éventuelles des facteurs taphonomiques et humains par rapport à l'agencement initial de la sépulture. À la différence près qu'il ne s'agit pas de déterminer les incidences du milieu de décomposition : les loculi sont des espaces vides retrouvés comme tels. Mais des éléments liés au corps ou des gestes funéraires, imperceptibles si l'attention n'est pas portée sur le squelette in situ, peuvent entraîner des distorsions dans l'agencement du squelette. Pour ce site, comme pour d'autres de la région alexandrine, les observations ostéo-archéologiques pallient la disparition de la matière organique (Georges et al., 2003) [Fig.9 et 10]. Les os, selon leur position (au sein de la sépulture et les uns par rapport aux autres), apportent en premier lieu des renseignements archéologiques sur la tombe, avant même de faire l'objet d'analyses paléobiologiques. Le corps est de facto replacé au cœur de la discussion, quelle que soit la richesse de la tombe dont l'étude s'avère globale. Grâce à une telle approche, nous avons révélé des éléments initialement placés sur ou autour du corps, mais aujourd'hui disparus.

Ce qui est valable pour les ossements l'est également pour les objets. L'analyse de mouvements éventuels du fait de la décomposition du corps contribue également à préciser le contexte de dépôt. Cette méthodologie permet aussi de discuter, sinon de la nature, tout du moins de la position de certains artéfacts initialement au contact du corps. C'est ainsi le cas des éléments de décoration de masques funéraires, seuls ceux étant parfaitement conservés dans les musées ayant fait l'objet d'études [Fig.11 et 12]. Ce qui a pu créer un biais typo-chronologique.
Adriani A., 1952, « Nécropole et ville de Plinthine », Annuaire du Musée gréco-romain, III, p. 140-159.

Boussac M.-F., 2001, « Deux villes de Maréotide : Taposiris et Plinthine », Bulletin de la Société française d'Égyptologie, 150, p. 42-72.

Boussac M.-F., 2005, «Taposiris Magna et Plinthine », in Archéologies. Vingt ans de recherches françaises dans le monde, Paris, Maisonneuve et Larose, p. 347.

Boussac M.-F., Georges P., Zouair N., 2006, « Égypte : un hypogée intact à Plinthine ", Archéologia, 431, p. 8-9.

Duday H., 2005, « L'archéothanatologie ou l'archéologie de la mort », in Dutour O., Hublin J.-J., Vandermeersch B. (ÉD), Objets et méthodes en paléoanthropologie, Comité des Travaux Historiques et Scientifique, Orientations et méthodes, n 7, Paris, p. 153-215.

Duday H., 2006, « L'archéothanatologie ou l'archéologie de la mort (Archaeoethnoanatology or the Archaeology of Death) ", in Gowland R., Knüssel C., Social Archaeology of Funerary Remains, Oxford, Oxbow books, p. 30-56.

Duday H., Guillon M., 2006, « Understanding the Circumstrances of Decomposition when the Body Is Skeletonized », in SснмітT A., Cunha E., Pinheiro J., Forensic Anthropology and Medecine. Complementary Sciences From Recovery to Cause of Death, Totowa, New Jersey, Humana Press, p. 116-157.

DunAND F., 2002, « De la cendre à la myrrhe. Les usages du corps mort en Égypte tardive », in LABRIQUE F. (ÉD.), Religions méditerranéennes et orientales dans l'Antiquité, Le Caire, Bibliothèque d'Étude, 135, p. 101-119.

Empereur J.-Y., NenNa M.-D. (ÉD.), 2001, Nécropolis 1, Études alexandrines 5, Le Caire, Ifao, 527p.

Empereur J.-Y., Nenna M.-D. (ÉD.), 2003, Nécropolis 2, Études alexandrines 7, Le Caire, Ifao, 714p.

Georges P., À paraître, « Où sont les enfants de la nécropole hellénistique de Plitnthine? ", in Types de tombes et traitement des enfants dans l'antiquité gréco-romaine, Actes du colloque d'Alexandrie 12-14 novembre 2009.

GeORGES P., 2002, « Les pratiques funéraires de la nécropole hellénistique de Plinthine : résultats préliminaires ", in CHARON A. (DIR.), La mort n'est pas une fin. Pratiques funéraires en Égypte d'Alexandre à Cléopâtre, Catalogue de l'exposition 28 septembre 2002-5 janvier 2003, Arles, Musée de l'Arles antique, p. 72-74.

Georges P., BoËs E., Alix G., Schmitt A., 2003, « Des momies éphémères et des os pour l'éternité. La gestion paradoxale de la Necropolis d'Alexandrie à l'époque romaine ", in BALLET P., Cordier P., Dieudonne-Glad N. (Dir.), La ville et ses déchets dans le monde romain : rebuts et recyclages, Actes du colloque de Poitiers (19-21 septembre 2002), Montagnac, Éditions Monique Mergoil, p. 277-301.

Maureil B., Sellier P., 1996, « Dislocation en ordre paradoxal, momification et décomposition : observations et hypothèses », in Castex D., Sellier P., Duday H., Bruzek J. (éd.), Méthodes d'étude des sépultures, du terrain à l'interprétation des ensembles funéraires, Paris, Bulletins et Mémoires de la Société d'Anthropologie de Paris, t. 8, n 3-4, p. 313-327.

Venit M., 2002, Monumental Tombs of Ancient Alexandria. The Theatre of the Dead, Cambridge, University press.

\section{Collaboration franco-israélienne dans la recherche archéologique}

Hamoudi Khalaily

Israel Antiquities Authority

$\mathrm{D}$ epuis le début du $\mathrm{xx}^{\mathrm{e}}$ siècle, lorsque la recherche archéologique en Israël a été établie, les délégations étrangères venues d'Europe et des États-Unis ont collaboré avec les institutions locales. Les savants français ont été des pionniers en ce domaine. René Neuville, par ses fouilles sur de nombreux sites néolithiques et mésolithiques, a été particulièrement actif dans le développement de l'archéologie israélienne. Ses études ont eu une influence majeure sur l'institutionnalisation de l'étude de la Préhistoire du sud du Levant et ses recherches coïncidaient avec celles de l'institut archéologique de l'Université hébraïque à Jérusalem. Son disciple et successeur, Jean Perrot, a créé la délégation permanente française à Jérusalem et a collaboré avec plusieurs 


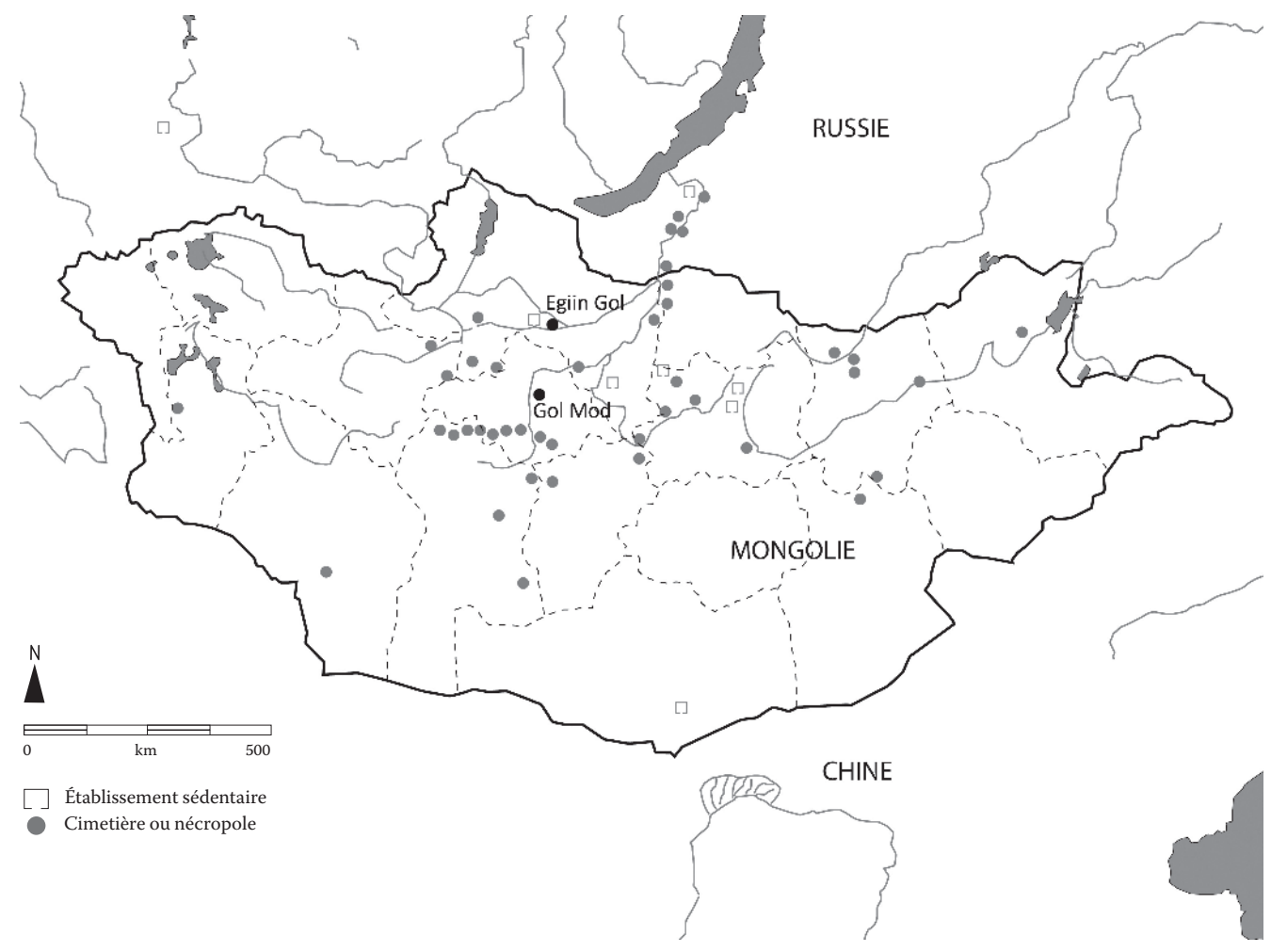

$\exists$

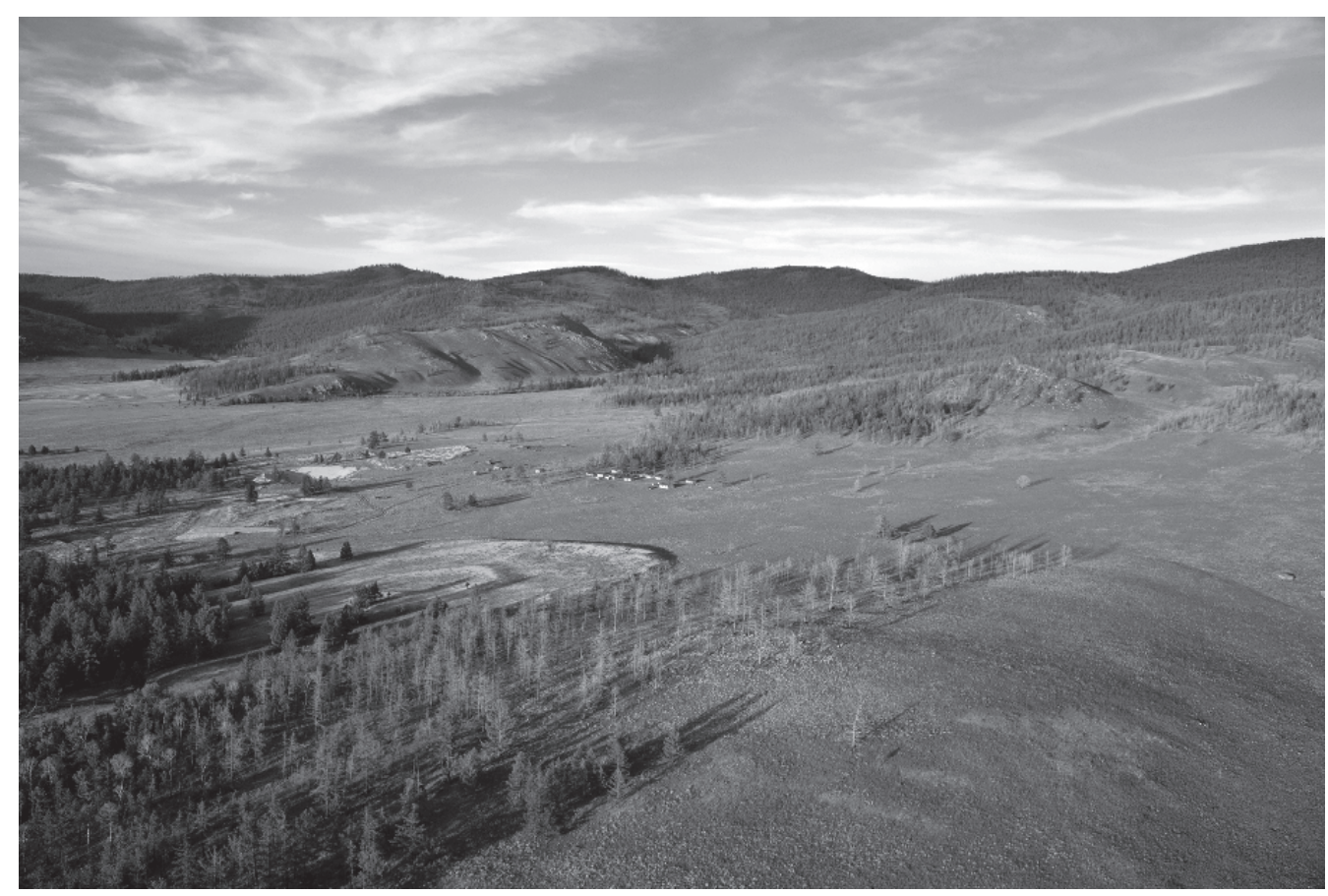

[Fig.1] Carte des sites archéologiques xiongnu de Mongolie.

[Fig.2] Vue aérienne du site de Gol Mod, Arkhangaï, Mongolie. 
institutions impliquées dans l'archéologie, comme l'Université hébraïque de Jérusalem, l'Université

Ben Gourion et l'Université de Tel-Aviv. Le centre français de Jérusalem (CRFJ) a accueilli de nombreux chercheurs français en archéologie et en anthropologie. Ils ont été intégrés à de nombreux projets sur le long terme, concernant diverses thématiques. Pierre de Miroschedgi a dirigé, avec l'Université hébraïque, la fouille de Tel Yarmouth, l'un des sites majeurs du Bronze ancien en Israël. François Valla a mené plusieurs projets avec différentes institutions (comme l'Université hébraïque de Jérusalem) sur la terrasse d'Hayonim et, avec l'Université Ben Gourion, une enquête préhistorique dans le nord du Néguev; plus récemment encore, il a participé aux fouilles de l'Ain Mallaha, en collaboration étroite avec l'Israel Antiquities Authority (IAA). Monique Lechevallier a fouillé le site de Hatula avec l'Université de Haïfa. Bernard Vandermeersch, Anne-Marie Tallier et d'autres chercheurs français ont travaillé sur les occupations de l'homme de Néandertal dans les grottes du Carmel et en Galilée, en collaboration avec les institutions israéliennes.

Le récent accord de coopération scientifique internationale entre l'IA A et l'Inrap a été une plateforme importante pour envisager une collaboration sur des projets archéologiques en Israël. Dans le cadre de cet accord, les chercheurs des deux instituts se doivent de «partager leurs compétences dans le domaine de l'archéologie préventive, de participer à des opérations archéologiques et de faciliter les collaborations scientifiques mutuelles dans les recherches archéologiques qu'ils effectuent ». Sur la base de cet accord, deux projets archéologiques ont été menés récemment en Israël: la reprise de l'étude du site de Beisamoun (cf. article p. 135) et le projet Césarée (cf. p. 103).

La fouille de Beisamoun est un projet codirigé par l'équipe d'experts de trois instituts : le CNRS, représenté par Fanny Bocquentin, l'IAA, par Hamoudi Khalaily, et l'Inrap, par Nicolas Samuelian. Le site de Beisamoun (9000-7900 avant notre ère) est situé dans la partie occidentale de la vallée de Hula, et ses vestiges s'étendent sur une superficie d'environ 12 ha. Le site a été sondé en 1965 par des archéologues français et les structures découvertes (murs, planchers en plâtre et éléments lithiques) ont permis de le dater du Néolithique précéramique B. Il n'y a plus eu d'investigations archéologiques de 1972 à 2007. Le projet actuel vise à étudier les techniques architecturales de la fin du Néolithique précéramique, à analyser systématiquement les assemblages de silex et de faune ; il offre aussi une occasion rare d'étudier, dans une perspective diachronique, la dynamique de l'occupation des sites d'exploitation de l'environnement et la transmission culturelle au sein du bassin de Hula. Les diverses compétences, expériences et méthodes de fouille des membres de l'équipe sont un atout majeur pour atteindre ces objectifs. Le projet n'en est qu'à ses débuts et les possibilités de découvertes sont nombreuses, comme le montrent les rapports des premières saisons. Nicolas Samuelian supervise l'une des deux zones de fouilles tout en étant responsable de l'enregistrement des données. C'est en s'appuyant sur son expérience qu'ont pu être menés un grand décapage suivant les méthodes développées par les archéologues de l'Inrap et une analyse stratigraphique très fine, deux réalisations qui contribuent à la compréhension des vestiges néolithiques.

La fouille à Césarée est un autre exemple d'une collaboration tripartite entre l'IAA, représenté par Hervé Barbé, l'Inrap, par Jocelyn Martineau et Fabien Sanz-Pascual, et l'Université de Nantes, sous la direction de Nicolas Faucherre. Il s'agit d'étudier l'architecture mamelouke du château, de la ville, du mur d'enceinte et du port. La responsabilité des archéologues de l'Inrap porte autant sur le relevé des vestiges archéologiques que sur leur étude et l'élaboration d'hypothèses de reconstitution.

Pour l'IAA, les recherches menées sur ces sites fondamentaux sont primordiales pour la compréhension des périodes préhistoriques et historiques en Israël. Cette collaboration est également précieuse car elle offre la possibilité aux diverses institutions partenaires de combiner leurs ressources et leurs connaissances pour la promotion de la science archéologique.

\section{Nouvelle approche par l'archéozoologie dans les nécropoles antiques d'Egiin Gol et de Gol Mod (Mongolie)}

\author{
Hélène Martin \\ Inrap, Archéozoologue et UMR 5608 " Traces» \\ Guilhem André \\ Mission archéologique française en Mongolie (MAFM)/ \\ Musée des Arts asiatiques-Guimet \\ Jean-Paul Desroches \\ MAFM/Musée des Arts asiatiques-Guimet
}

P résupposés sur la civilisation xiongnu. La connaissance de la civilisation xiongnu ( $\mathrm{III}^{\mathrm{e}}$ siècle avant notre ère - $\mathrm{III}^{\mathrm{e}}$ de notre ère) de Mongolie s'est longtem ps fondée sur les informations textuelles émanant de leurs voisins chinois, chroniqueurs de la dynastie des Han (206 avant notre ère-220 de notre ère) et des suivantes. Les annales historiques de l'Empire constituent en effet des sources primordiales, mais l'objectivité de certains auteurs antiques demeure aléatoire, de même que la fiabilité de leurs informations, eut égard aux relations conflictuelles entre ces deux puissances voisines : les lettrés se trouvaient confrontés à des problèmes d'accès aux données, de transcription et de transmission de leurs textes.

Pour une majorité de spécialistes, l'organisation des Xiongnu adoptait la forme d'une confédération de tribus occupant un territoire qui s'étendait, à l'apogée de leur puissance, de l'Altaï à la Mandchourie et du Baïkal à la boucle du fleuve Jaune [Fig.1]. À la tête de cette confédération, leurs 